\title{
Design of a Database-Driven GPC for Nonlinear Systems
}

\author{
Zhe Guan \\ KOBELCO Construction Machinery Dream-Driven Co-Creation Research Center, Hiroshima University \\ Higashi-Hiroshima, 739-8527, Japan \\ E-mail: guanzhe@hiroshima-u.ac.jp, \\ Toru Yamamoto \\ Graduate School of Advanced Science and Engineering, Hiroshima University \\ Higashi-Hiroshima, 739-8527, Japan \\ E-mail: yama@hiroshima-u.ac.jp
}

\begin{abstract}
This paper presents a Generalized Predictive Control (GPC) design scheme based on database-driven approach for nonlinear systems. In industrial systems, lots of controlled plants with unknown time-delay and strong nonlinearity, are difficult to be handled in terms of control performance. The databased-driven approach has been attracted attention to tackle non-linearity issue without system information. On the other hand, GPC is considered in cases where the time-delay is unknown. The proposed scheme is validated through a numerical simulation.
\end{abstract}

Keywords: GPC, Database-Driven approach, Nonlinear Systems

\section{Introduction}

Industrial process control is a large and diverse field, and its broad range of applications require a wide range of controllers including model predictive control (MPC) and a variety of nonlinear controllers in order to maintain system performance. Among them, the Generalized Predictive Control (GPC) algorithm ${ }^{1}$ can adapt to process time-delay and model order based on the long-range prediction. As a result, it is shown to be particularly effective for the self-tuning control of industrial processes $^{2}$, in which are widely applied. However, regarding to the GPC, the excellent control behavior can be achieved, provided that the number of parameters in the model are required. It is important to include a reasonable representation of disturbances and other unknown dynamics, such that the correct controller can be designed. In other words, the GPC is model-based technique, so good performance needs a high-quality process model. Unfortunately, as it is well known, modeling for an industrial process using first principles or identification is a difficult task. Further, even the mathematical model of the industrial process is established, it only approximates the process but not involves entire process dynamics. Moreover, it is timeand cost-consuming to build the relative accurate complicate model of industrial process. Therefore, some challenges have been addressed in the model-based technique.

With the rapid development of the computer technology, industrial processes have undergone significant changes. It is possible to store and process huge amounts of process data with the help of computers. In recent years, it is a trend to design the controller by directly using online or offline Input/Output (I/O) data obtained from the controlled system or knowledge, instead of the explicit mathematical model of the controlled process $^{3}$. Several methods ${ }^{4,5}$ have been 
proposed by considering the above idea. Moreover, the data-oriented GPC controller ${ }^{6}$ has been proposed, where the initial control parameters are estimated from the I/O data, and then the corresponding polynomials are calculated. However, two issues are still not addressed. The aforementioned method is not able to deal with some systems with nonlinearity. The other issue is that the tuning performs in off-line manner.

The database-driven approach ${ }^{7}$ is one of control strategies applied in systems with non-linearity since it can tune the control parameters rapidly at each equilibrium point. Furthermore, the database-driven approach performs the tuning in on-line manner in order to deal with the operation condition along the time, such that the performance deterioration is able to be avoided. Successful implementations of the data-driven approach have been presented in the technical literatures ${ }^{8,9,10}$.

Based on the above observation, this paper newly presents a design scheme that the controller is designed with GPC algorithm based on database-driven approach. The proposed scheme inherits the advantages of GPC algorithm in terms of handling delay. Moreover, the control parameters are computed on-line in a local bounded neighbor data which is extracted from the database.

\section{Problem Statement}

Consider the following nonlinear system:

$$
y(t)=f(\phi(t-1))+\xi(t)
$$

where $y(t), f(\cdot)$ and $\xi(t)$ denote the system output, the nonlinear function and the white Gaussian noise with zeros mean and variance $\sigma^{2}$, respectively. Additionally, $\phi(t-1)$ is called information vector, which is defined as follows:

$$
\begin{aligned}
\phi(t-1):= & {\left[y(t-1), \cdots, y\left(t-n_{y}\right),\right.} \\
& \left.u(t-d-1), \cdots, u\left(t-d-n_{u}\right)\right],
\end{aligned}
$$

where $u(t)$ and $d$ represent the control input and system delay time. Also, $n_{y}$ and $n_{u}$ are the order of the system output and control input, respectively.

When considering regulation about a particular operation point, it is reasonable to assume that the nonlinear system can be described as a locally-linearized model by the following equation:

$$
A\left(z^{-1}\right) y(t)=z^{-(d+1)} B\left(z^{-1}\right) u(t)+\frac{\xi(t)}{\Delta},
$$

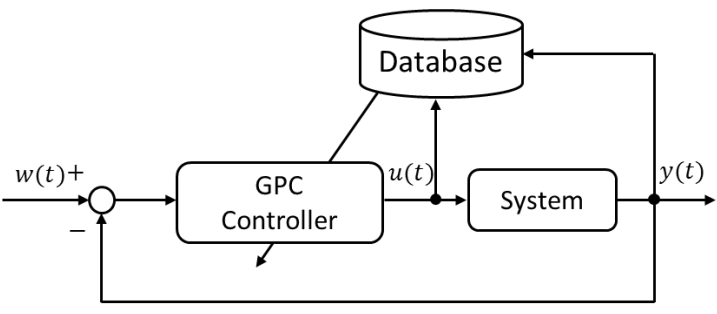

Fig. 1. Block diagram of the proposed scheme.

where $\mathrm{z}^{-1}$ represents the backward shift operator which implies $\mathrm{z}^{-1} y(t)=y(t-1)$, and $\Delta$ denotes the differential operator defined by $\Delta:=1-\mathrm{z}^{-1} \cdot A\left(\mathrm{z}^{-1}\right)$ and $B\left(z^{-1}\right)$ are the polynomials which are formulated as follows.

$$
\begin{aligned}
& A\left(z^{-1}\right)=1+a_{1} z^{-1}+\cdots+a_{n_{y}} z^{-n_{y}}, \\
& B\left(z^{-1}\right)=b_{0}+b_{1} z^{-1}+\cdots+a_{n_{u}} z^{-n_{u}} .
\end{aligned}
$$

Based on the above statement, the objective of this study is to design a GPC controller that generates $u(t)$ to controlling the system Eq. (1) based on database-driven approach such that the output can track the reference value. As a result, the block diagram of the proposed scheme is depicted in Fig. 1.

\section{Proposed Scheme Design}

\subsection{Generalized Predictive Control}

The GPC law is derived based on the minimization of the criterion in the following equation:

$$
\begin{aligned}
J= & E\left[\sum_{j=1}^{N}\{y(t+d+j)-w(t+d+j)\}^{2}\right. \\
& \left.+\sum_{j=1}^{N} \lambda(j)\{\Delta u(t+j-1)\}^{2}\right],
\end{aligned}
$$

where $E(\cdot)$ is the expectation value and $[1, \mathrm{~N}]$ denotes the range of control horizon and prediction horizon. $w(t)$ is the reference value and $\lambda(j)$ is a user-specified control weighting sequence. The control law based on Eq. (6) is

$$
\begin{aligned}
& \underset{j=1}{\operatorname{derivad}{ }^{N} p_{j} F_{j}\left(z^{-1}\right) y(t+d)+}+\left\{1+z^{-1} \sum_{j=1}^{N} p_{j} S_{j}\left(z^{-1}\right)\right\} \Delta u(t) \\
& -\sum_{j=1}^{N} p_{j} w(t+d+j)=0,
\end{aligned}
$$

with $F_{j}\left(z^{-1}\right)$ and $G_{j}\left(z^{-1}\right)$ are derived by the following Diophantine equations:

(C) The 2021 International Conference on Artificial Life and Robotics (ICAROB2021), January 21 to 24, 2021 


$$
\begin{array}{r}
1=\Delta A\left(z^{-1}\right) E_{j}\left(z^{-1}\right)+z^{-j} F_{j}\left(z^{-1}\right) \\
E_{j}\left(z^{-1}\right) B\left(z^{-1}\right)=G_{j}\left(z^{-1}\right)+z^{-1} S_{j}\left(z^{-1}\right)
\end{array}
$$

where

$$
\begin{aligned}
& E_{j}\left(z^{-1}\right)=1+e_{1} z^{-1}+\cdots+e_{j-1} z^{-(j-1)} \\
& F_{j}\left(z^{-1}\right)=f_{0}^{j}+f_{1}^{j} z^{-1}+\cdots+f_{n_{y}}^{j} z^{-n_{y}} \\
& G_{j}\left(z^{-1}\right)=g_{0}+g_{1} z^{-1}+\cdots+g_{j-1} z^{-(j-1)} \\
& S_{j}\left(z^{-1}\right)=s_{0}^{j}+s_{1}^{j} z^{-1}+\cdots+s_{n_{u}-1}^{j} z^{-\left(n_{u}-1\right)}
\end{aligned}
$$

Moreover, $p_{j}$ is formulated by

$$
\left[p_{1}, p_{2}, \cdots, p_{N}\right]:=[1,0, \cdots, 0]\left[\boldsymbol{G}^{T} \boldsymbol{G}+\Lambda\right]^{-1} \boldsymbol{G}^{T}
$$

with the matrix $\boldsymbol{G}$ is composed by

$$
\boldsymbol{G}:=\left[\begin{array}{cccc}
g_{0} & 0 & & \\
g_{1} & g_{0} & 0 & \\
\vdots & \vdots & \ddots & \\
g_{N-1} & g_{N-2} & \ldots & g_{0}
\end{array}\right]
$$

\subsection{Derivation of output by control parameters}

Considering the Diophantine equations Eq. (8) and substitute to system Eq. (3), the system output can be obtained one-step ahead as:

$$
\hat{y}(t+1)=F_{1} y(t)+G_{1} \Delta u(t-d)
$$

The above equation is written in the form of information vector as:

$$
\hat{y}(t+1)=\phi^{T}(t) \theta(t)
$$

with $F_{1}$ and $G_{1}$ are the components in $\theta(t)$.

Once the control parameters $F_{1}$ and $G_{1}$ are identified, and then by recursive formula, the control signal is able to be derived. Please refer to the Ref. 6.

\subsection{Database-driven approach}

The detail of this approach will not be given because of pages limitation. Please refer to the Ref. 7 . We will give core part in terms of control parameters update. As mentioned in Eq. (13), the control parameters will be updated based on database by adopting the steepest descent method.

$$
\theta^{\text {new }}(t)=\theta^{\text {old }}(t)-\eta \frac{\partial J(t+1)}{\partial \theta(t)}
$$

with $\eta$ learning rate, and $J(t+1)$ is defined by:

$$
J(t+1):=\frac{1}{2} \varepsilon(t+1)^{2}
$$
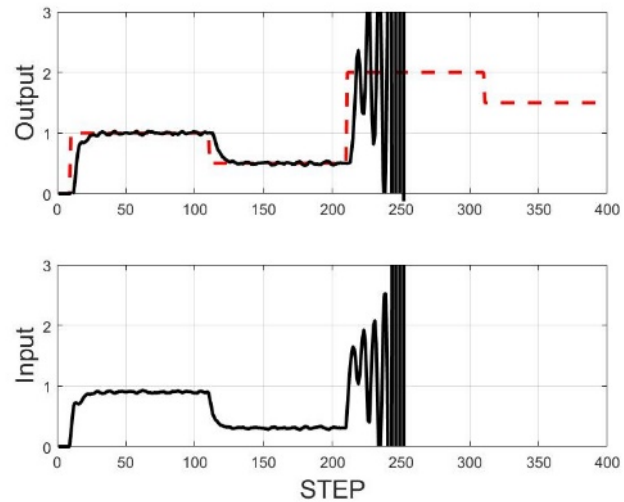

Fig. 2. Control results using the conventional PID control.
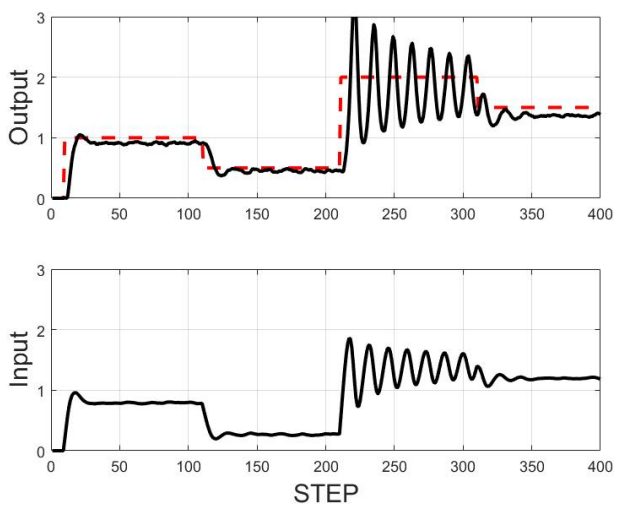

Fig. 3. Control results using the data-oriented GPC.

\section{Numerical Simulation}

In this section, we have conducted the simulation and illustrate the effectiveness of the proposed scheme. The Hammerstein model ${ }^{11}$ is considered and is given in the following form:

$$
\begin{aligned}
y(t)= & 0.6 y(t-1)-0.1 y(t-2) \\
& +1.2 x(t-d-1)-0.1 x(t-d-2)+\xi(t) \\
x(t)= & u(t)-u^{2}(t)+0.5 u^{3}(t)
\end{aligned}
$$

where $\xi(t)$ is the Gaussian noise with zero mean and variance $0.01^{2}$, and delay time $d$ is set as 2 in this study. The reference signals are given by:

$$
w(t)=\left\{\begin{array}{l}
1.0(0 \leq t<100) \\
0.5(100 \leq t<200) \\
2(200 \leq t<300) \\
1.5(300 \leq t<400)
\end{array}\right.
$$



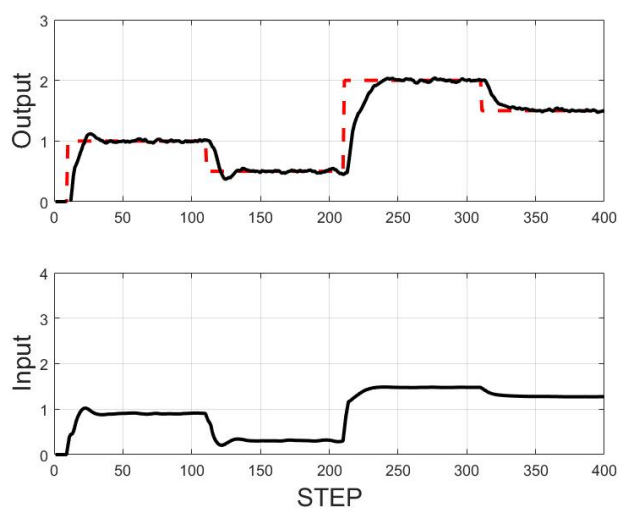

Fig. 4. Control results using the proposed scheme. Additionally, the user-specified parameters involved in the proposed scheme are determined as shown in Table 1.

Table 1. The user-specified parameters.

\begin{tabular}{|l|c|}
\hline The orders of information vector & $\begin{array}{l}n_{y}=2 \\
n_{u}=5\end{array}$ \\
\hline Numbers of Neighbors & $k=5$ \\
\hline Weighting factor & $\lambda=15$ \\
\hline Initial data-base $N(0)$ & 20 \\
\hline Learning rate & 100 \\
\hline
\end{tabular}

The proposed scheme cannot work unless the initial database is generated. Consequently, the conventional Proportional-Integral-Derivative (PID) control tuned by the Chien-Hrones-Reswick method ${ }^{12}$ is employed. The related parameters are shown as:

$$
K_{P}=0.486, K_{I}=0.227, K_{D}=0.122 .
$$

The control results are depicted in Fig. 2, where the output is become unstable after 200 [STEP] because of nonlinearity and delay time. These I/O data form the basis of the initial database. Fig. 3 shows the control results where the control parameters $F_{1}$ and $G_{1}$ in GPC algorithm are identified from the I/O data and are identical in the control process. The tracking property is poor, and oscillation occurs after 200 [STEP]. Finally, the proposed scheme is employed to the system, and Fig. 4 shows that the proposed scheme outperforms the ones in Fig. 3. It should be noted that the control parameters $F_{1}$ and $G_{1}$ are updated based on database along the time.

\section{Conclusions}

In this paper, the design of a data-driven GPC control for nonlinear systems has been presented. First, according to the proposed scheme, the model information is not required in designing the controller. Then, the databasedriven approach is considered to deal with systems with strong non-linearity and provides a way for the online utilization of the proposed scheme. Furthermore, the proposed scheme inherits the advantage of GPC algorithm such that good performance can be achieved regardless of time-delay. Finally, the effectiveness has been verified on a numerical simulation. The experimental evaluation will be in our future work.

\section{References}

1. D. W. Clarke, C. Mohtadi, and P S. Tuffs, Generalized Predictive Control - Part I. The basis algorithm, Automatica, 23(2), 1987, pp. 137-148.

2. D. W. Clarke, Application of generalized predictive control to industrial processes, IEEE Control Systems Magazine. 8(2), 1988, pp. 49-55.

3. Z. S. Hou and S. S. Xiong, On Model-Free Adaptive Control and its Stability Analysis, IEEE Transactions on Automatic Control, 64(11), 2019, pp. 4555-4569.

4. M. C. Campi, A. Lecchini, and S. M. Savaresi, Virtual reference feedback turning: a direct method for the design of feedback controllers, Automatica, 38(8), 2002, pp 1337-1346.

5. H. Hjalmarsson, M. Gevers, S. Gunnarsson, and O. Lequin, Iterative feedback tuning: theory and applications, IEEE Control Systems Magazine, 18(4), 1998, pp. 26-41.

6. Z. Guan, S. Wakitani, and T. Yamamoto, Design and Experimental Evaluation of a Data-Oriented Generalized Predictive PID Controller, Journal of Robotics and Mechatronics, 28(5), 2016, pp. 722-729.

7. T. Yamamoto, K. Takao, and T. Yamada, Design of a Data-Driven PID control, IEEE Transactions on Control Systems Technology, 17(1), 2009, pp. 29-39.

8. K. Takao, T. Yamamoto, and T. Hinamoto, A new GPCbased PID controller using memory-based identification, Proceedings of the 11th IFAC International Workshop on Adaptation and Learning in Control and Signal Processing, 2013, pp. 587-592.

9. S. Wakitani, M. C. Deng, and T. Yamamoto, Design of a data-driven controller for a spiral heat exchanger, Proceedings of 11th IFAC Symposium on Dynamics and Control of Process Systems Including Biosystems, 49(7), 2016, pp. 342-346.

10. Z. Guan, I. Mizumoto, and T. Yamamoto, Design of PCAbased Data-Driven Adaptive Output Feedback Control for Nonlinear Systems, IEEE 28th International Symposium on Industrial Electronics, 2019, pp. 497-502.

11. Z. Q. L, On identification of the controlled plants described by the Hammerstein system, IEEE Transactions on Automatic Control, 39(2), 1994, pp. 569-573.

12. K. L. Chien, J. A. Hrones, and J. B. Reswick, On the automatic control of generalized passive systems, Transaction ASME, 74, 1972, pp. 175-185. 